\title{
Testosterone undecanoate - increase of oral bioavailability by nanostructured lipid carriers (NLC)
}

\author{
M. Muchow ${ }^{1,2}$, P. Maincent ${ }^{2}$, R. H. Müller ${ }^{1}$ and C. M. Keck ${ }^{1,3 *}$ \\ *Correspondence: ck@ckc-berlin.de \\ ${ }^{1}$ Freie Universität Berlin, Department of Pharmacy, Pharmaceutical Technology, Biopharmaceutics and NutriCosmetics, Kelchstr. 31 , 12169 \\ Berlin/Germany. \\ 2Université de Lorraine, Laboratory of Pharmaceutical Technology and Biopharmacy, 5, rue A. Lebrun, 54000 Nancy/France. \\ 3University of Applied Sciences Kaiserslautern, Applied Pharmacy Division, Campus Pirmasens, Carl-Schurz-Str. 10-16, 66953 Pirmasens/ \\ Germany.
}

\begin{abstract}
Testosterone undecanoate (TU) has a low oral bioavailability of about $3 \%$ in beagle dogs and $7 \%$ in men. To avoid administration of a single dose in two oral units and to increase lymphatic absorption to minimise side effects in the liver, TU was incorporated into nanostructured lipid carriers (NLC) and formulated as drug nanocrystals. Testosterone (T) was run as nanocrystal formulation for reasons of comparison. NLC and drug nanocrystals were produced by high pressure homogenisation. The in vivo studies were performed in male Wistar rats. The drug absorbed was quantified as testosterone analysed by EIA assay. The nanocarriers were compared with the commercial product Andriol Testocaps. The drug nanocrystals reached about half the AUC of Andriol Testocaps ${ }^{\varpi}$ (testosterone) or were just below (testosterone undecanoate). The best NLC formulations possessed a 2 times higher AUC compared to Andriol Testocaps ${ }^{\oplus}$. Highest bioavailability was obtained with $200 \mathrm{~nm}$ NLC. Nature of the lipid for the particle matrix and the drug loading (15\%, $30 \%)$ affected distinctly the shape of the serum profile $\left(c_{\max }, t_{\max }\right.$, prolongation of serum levels). Modification of these parameters should allow controlled adjustment of the serum profile to therapeutic needs. Due to the reduced volume of the nanocarriers, application of a single dose in one unit is possible.
\end{abstract}

Keywords: Nanosuspensions, lipid nanoparticles, testosterone, testosterone undecanoate, bioavailability enhancement

\section{Introduction}

Testosterone $(T)$ has a poor bioavailability due to its extensive first pass metabolism by the liver. Therefore in commercial products lipidic derivatives of testosterone are used, e. $g$. testosterone undecanoate (TU) and testosterone enantate (TE). Still the oral bioavailability (BA) is very low, approx. about $7 \%$ for TU in men and $3 \%$ in beagle dogs $[1,2]$. The discontinued product Andriol ${ }^{\circledast}$ was a solution of TU in oleic acid [1]. The successor commercial product Andriol Testocaps ${ }^{\circledR}$ is a solution of TU in castor oil and lauroglycol FCC [2]. The rationale for replacing Andrio $^{\circledast}$ by Andriol Testocaps ${ }^{\circledast}$ was the low stability and the very short shelf life (3 months) of the original product. It had to be stored at $8^{\circ} \mathrm{C}$. Due to the low solubility of TU in the oil a single dose of $80 \mathrm{mg}$ has to be administered in two capsules.

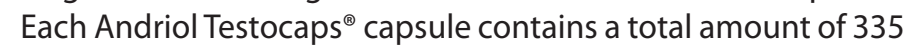
mg TU solution. Taking two capsules is not very convenient for the patient and reduces compliance. Therefore the aim was to develop a formulation which allows administration in a single oral unit. In addition the bioavailability should be increased to minimise undesirable drug effects. The bioavailable TU is mainly absorbed via the lymphatic route [1-5]. Minor parts of TU are immediately de-esterified to testosterone at the gut wall. The major part is still absorbed across the gut wall but then immediately metabolised in the liver (first pass) $[1,3,4]$. This unavailable amount for therapy creates stress to the liver $[6,7]$. Therefore an additional aim was to increase the fraction absorbed by the lymphatic route. Achievement of this goal can be seen when the bioavailability increases, because almost all the TU detectable in the blood is due to lymphatic absorption.

The approach taken was to incorporate TU into nanostructured lipid carriers (NLC). NLC are lipid nanoparticles made from a blend of a solid lipid and a liquid lipid (oil) which is solid at body temperature $[8,9]$. NLC have several advantages compared to the first generation of lipid nanoparticles, the solid lipid nanoparticles (SLN) [9-13]. For the poorly soluble and poorly orally bioavailable drug cyclosporine it could be shown that SLN increase the bioavailability leading to a serum concentration being similarly long in the therapeutic range - identical to the microemulsion Sandimmun Neoral - but avoiding the serum peak held responsible for nephrotoxicity [14-18]. Therefore the lipid nanoparticles were chosen as first choice nanocarrier system, of course using the second generation NLC. Various formulation parameters were investigated in in vivo studies regarding their influence on the bioavailability: Particle size of the NLC, nature of the lipid (glyceride versus fatty acid) and drug loading of the particles (30\% versus $15 \%$ ). 
Another promising approach to increase the oral bioavailability of poorly soluble drugs are drug nanocrystals [19-22]. Production of drug nanocrystals is simpler than producing NLC. Therefore and for reasons of convenience and costs, drug nanocrystals can be considered as carrier system of first choice. In case the drug nanocrystals do not work or do not work sufficiently, the NLC are the alternative delivery system. NLC are especially of value when the presence of lipid promotes drug absorption. This is the case for TU because the bioavailability is very much dependent on the fed state $[23,24]$. Considering this, NLC were chosen as nanocarrier system. However, it appeared also worthwhile to run nanocrystals formulations as well.

Not only TU was investigated, also the "original" molecule testosterone. After absorption TU is fast metabolised into T, therefore it was a logic approach by companies to use TU instead of T because of its higher bioavailability. Nevertheless there is the general trend in pharmacy to use rather original molecules than synthetic derivatives. For this reason testosterone was also included. In addition, testosterone nanocrystals could contribute to better understanding of mechanisms and be used for exploring new potential opportunities. Furthermore, testosterone could not be formulated as NLC since its solubility in lipid mixtures is too low.

\section{Materials and Methods Materials}

Tesotsterone undecanoate was bought from Jenapharm (Jena, Germany), testosterone from Caelo (Hilden, Germany). For the production of the lipid nanoparticles the following lipids and surfactants were used: Dynasan 118 was obtained from Condea (Witten, Germany). Stearic acid, sodium dodecylsulfate and Tween 80 were bought from Sigma-Aldrich (Deisenhofen, Germany), oleic acid from Caelo. All excipients were used as received.

\section{Methods}

\section{Nanostructured lipid carriers (NLC)}

NLC were produced by high pressure homogenization. The solid lipid (Dynasan 118 or stearic acid) was heated to a temperature of $10^{\circ} \mathrm{C}$ above its melting point. Oleic acid was admixed to the melted lipid. The active ingredient was then dissolved in the lipid blend. A pre-emulsion was prepared by pouring the lipid phase into an aqueous Tween 80 solution ( $1 \%$ or $2 \%$ ) of the same temperature and mixing 30 seconds with an Ultra Turrax T-25 basic with an S 25 N - 10 G dispersing element (Janke \& Kunkel GmbH, Staufen, Germany). The obtained crude pre-emulsion was then homogenized 5 times at $80^{\circ} \mathrm{C}$ with an Avestin Emulsiflex B3 homogenizer (Avestin Europe, Mannheim, Germany) which was equipped with a heating system. A homogenization pressure of approx. 200 to 800 bars was applied depending on the desired particle size. Formulations where then cooled in glass vials to room temperature. An overview of all formulations is given in table 1.
Table 1. Overview of the formulations used for the in vivo tests. All NLC formulations contained a $10 \%$ lipid phase (lipid+drug) dispersed in a Tween 80 solution of $1 \%$ or $2 \%$.

\begin{tabular}{|c|c|}
\hline Formulation & Composition \\
\hline $\begin{array}{l}\text { Andriol in Tween } 80 \\
\text { solution }\end{array}$ & $\begin{array}{l}25 \mathrm{mg} \text { TU solution from Andriol Testocaps cap- } \\
\text { sule dispersed in } 500 \mu \mathrm{l} \text { Tween } 80 \text { solution } 0.1 \%\end{array}$ \\
\hline $\begin{array}{l}\text { Andriol in Castor Oil/ } \\
\text { Lauroglycol }\end{array}$ & $\begin{array}{l}\text { solution from Andriol Testocaps capsule diluted } \\
\text { to } 1 \% \text { TU content with castor oil and Laurogly- } \\
\text { col FCC }\end{array}$ \\
\hline TU Nanosuspension & $1 \% \mathrm{TU}$ in $0.2 \%$ sodium dodecylsulfat solution \\
\hline T Nanosuspension & $1 \% \mathrm{~T}$ in $0.2 \%$ sodium dodecylsulfat solution \\
\hline $\begin{array}{l}\text { NLC } 30 \% \text { Dynasan } \\
\text { (approx. } 200 \mathrm{~nm} \text { ) }\end{array}$ & $\begin{array}{l}30 \% \text { TU in Dynasan } 118 / \text { oleic acid }(50 / 50) \text { in } \\
2 \% \text { Tween } 80 \text { solution }\end{array}$ \\
\hline $\begin{array}{l}\text { NLC } 30 \% \text { Dynasan } \\
\text { (approx. } 600 \mathrm{~nm} \text { ) }\end{array}$ & $\begin{array}{l}30 \% \text { TU in Dynasan } 118 / \text { oleic acid }(50 / 50) \text { in } \\
1 \% \text { Tween } 80 \text { solution }\end{array}$ \\
\hline $\begin{array}{l}\text { NLC } 15 \% \text { Dynasan } \\
\text { (approx. } 200 \mathrm{~nm} \text { ) }\end{array}$ & $\begin{array}{l}15 \% \text { TU in Dynasan } 118 / \text { oleic acid }(50 / 50) \text { in } \\
2 \% \text { Tween } 80 \text { solution }\end{array}$ \\
\hline $\begin{array}{l}\text { NLC } 15 \% \text { stearic acid } \\
\text { (approx. } 200 \mathrm{~nm} \text { ) }\end{array}$ & $\begin{array}{l}15 \% \mathrm{TU} \text { in stearic acid/oleic acid (50/50) in } 2 \% \\
\text { Tween } 80 \text { solution }\end{array}$ \\
\hline
\end{tabular}

Particle sizes were then analysed by photon correlation spectroscopy (PCS) using a Zetasizer $2000 \mathrm{HS}$ and by Laser Diffractometry (LD) using a Mastersizer X (both Malvern Instruments, Malvern, UK). Zeta potential was assessed also using the Zetasizer $2000 \mathrm{HS}$.

\section{Nanosuspensions}

Nanosuspensions where prepared by ball milling the bulk product with a Bühler PML-2 mill (Bühler, Uzwil, Switzerland) for 30 minutes with pearls of $0.2 \mathrm{~mm}$. The obtained crude suspension was then homogenized with an LAB 40 homogenizer (APV Deutschland GmbH, Unna, Germany) with 3 cycles at 1000 bars and 2 cycles at 1500 bars at room temperature. Particle sizes were measured as mentioned above.

\section{In vivo experiments}

Experiments were carried out in compliance with the French legislation on animal experiments under the personal experimentation authorization No. 54-68. Animals were deprived of food 12 hours before the treatment but still had free access to water. Prior to administration of the oral dose, a blood sample was taken to determine the testosterone level before the treatment.

Rats were divided into three groups of four animals and one replacement animal. The replacement animal was treated exactly the same way as the other rats and was used in case of one of the "regular" group members could not be used. The freshly prepared samples were administered to the rats by means of a feeding needle and a syringe. The dose applied was $10 \mathrm{mg}$ TU per $\mathrm{kg}$ body weight. In case of the administration of testosterone the dose was adapted to be equal to the TU value (i.e. $6.32 \mathrm{mg} \mathrm{T}$ per $\mathrm{kg}$ body weight). The volume of administration was typically between 250 and 
$500 \mu \mathrm{l}$ depending on the weight of the animal. The dead volume of the feeding needle was determined for every sample to guarantee the exact dose to be delivered into the stomach of the rat. After administration, rats were put back into the cages where they were allowed to move freely and had access to drinking water.

Blood samples were drawn prior to the administration to determine the basal testosterone serum value and after 1,2 , $3,4,6$ and 8 hours past the administration by drawing $400 \mu \mathrm{l}$ of blood from the animal. After 30 minutes of cold storage $\left(4^{\circ} \mathrm{C}\right.$ ) the samples were centrifuged at $6,000 \mathrm{~g}$ (also at $4^{\circ} \mathrm{C}$ ).

The obtained serum was then immediately transferred into polypropylene tubes which were stored at $-80^{\circ} \mathrm{C}$ until they were assayed for testosterone concentration.

Serum testosterone levels and pharmacokinetic values Serum testosterone levels were assessed using an Enzyme immuno assay (EIA) test kit purchased from Cayman Chemicals (Ann Arbor, Michigan, USA). The analysis was done using the "cold spike extraction" protocol supplied by the manufacturer. Briefly, a fraction of the samples were spiked with testosterone. An extraction with diethyl ether under a nitrogene stream was then performed. Both spiked and unspiked samples were analysed in duplicate to determine the recovery rate. The other samples were analyzed unspiked assuming the found recovery rate to be valid for all samples. The plate was read using a Sunrise microplate reader at $405 \mathrm{~nm}$ (Tecan, Männedorf, Switzerland). Testosterone concentrations are calculated using the spreadsheet template which is part of the test kit. Basal values were subtracted from the measured concentrations. The area under the curve (AUC) of the concentration-time profile was calculated with the linear trapezoidal method.

\section{Results and Discussion}

\section{Bioavailability study of TU and T versus Andriol} Testocaps ${ }^{\circledast}$

Nanocrystals are a much simpler approach than NLC for the oral delivery of poorly soluble drugs. They can be considered as a formulation of first choice. Therefore in the first part of this in vivo study, nanocrystals of testosterone undecanoate (TU) and testosterone $(T)$ were prepared and compared regarding their bioavailability (BA) relative to Andriol Testocaps ${ }^{\circledR}$. In general, the commercial pharmaceutical products for testosterone replacement therapy do not contain the original molecule testosterone, but lipophilic derivatives, such as testosterone undecanoate (TU) and testosterone propionate (TP). The reason for this is very simple: The bioavailability of testosterone is even much lower than of the lipophilic derivatives. TU has an oral bioavailability of about $7 \%$, TP much below that [1]. In addition, TU is very fast de- esterified to mainly $T$ after absorption. Therefore in these in vivo studies, only T was analysed in the blood, not TU itself.

For reasons of market differentiation, there is still a demand of pharmaceutical industry to successfully deliver oral

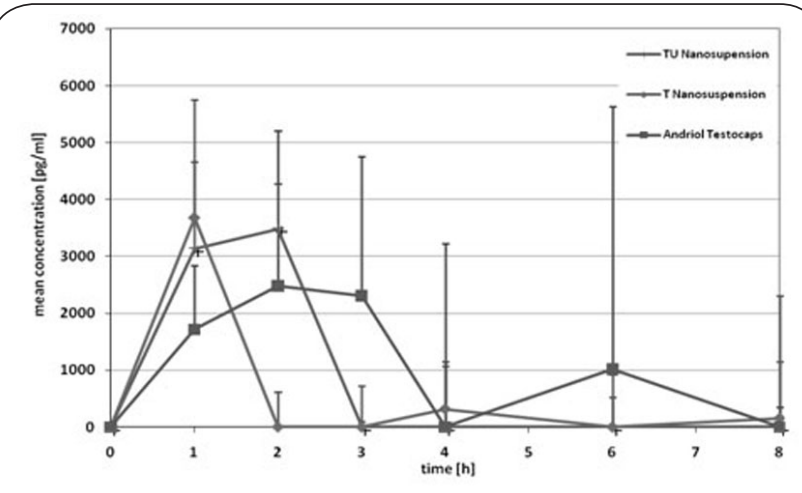

AUC values after 8 hours

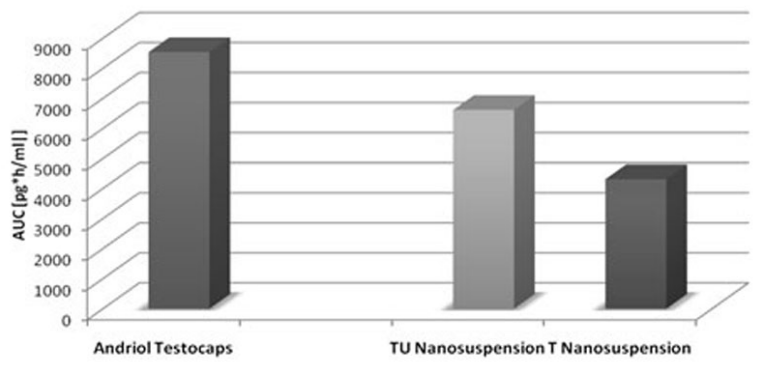

Figure 1. Testosterone serum concentration plot after an oral administration of T nanosuspension, TU nanosuspension and Andriol Testocaps dispersed in Tween 80 (upper).

testosterone. That is why for this study nanocrystals of both TU and T were prepared as described in the methods section. The size of the T nanocrystals was about $800 \mathrm{~nm}$. Further reduction by high pressure homogenisation was not possible since the material proved to be very hard. However, this size was sufficient for a first study for getting an impression how effective nanocrystal $T$ formulations can be. TU nanocrystals were prepared with a size of $474 \mathrm{~nm}$. The serum profiles show a very steep increase for both nanocrystal formulations at the first measuring point of one hour. The serum concentration is about double as high compared to Andriol Testocaps ${ }^{\oplus}$ (figure 1, upper). This is very interesting because:

1. It proves that absorption from the nanonised material is very fast.

2. Absorption of the 20 times larger sized T nanocrystals $(865 \mathrm{~nm})$ is as fast as the one for the small sized TU nanocrystals ( $474 \mathrm{~nm}$ ).

3. It cannot be excluded that especially for $T$ nanocrystals an even higher serum concentration is present at 0.5 hours (concluded from the fast decrease in serum concentration from one hour to two hours).

4. The fast decay of the T serum concentration is potentially attributed to the faster transit of the large nanocrystals through the gastrointestinal tract; the absorption window in the upper gut is passed faster.

5. The very small TU nanocrystals are more mucoadhesive, 
consequently they remain for a longer time in the absorption window.

6 . It is very remarkable that such high serum concentrations were obtained despite the fact that no lipid at all was co-administered with the nanocrystals, considering that lipids are known to increase the TU absorption.

Based on these data conclusions can be made for optimising the nanocrystal formulations:

1. The T nanocrystals dissolve fast, but measures need to be taken to keep them longer in the absorption window. 2. Increased retention time can be achieved by reducing the size or alternatively stabilising them with a mucoadhesive polymer (e. g. chitosan $\mathrm{HCl}$ ).

3. Lipid can be co-administered with the nanocrystals to exploit the absorption enhancing effect of lipids, thus further increasing the BA.

In the commercial product Andriol Testocaps ${ }^{\circledR}$, TU is dissolved in oil. Based on the consideration that administering $10 \mathrm{mg}$ TU per kg bodyweight will lead to serum concentrations of T which can be analytically quantified, this dose was chosen for all in vivo tests. Administering this oil solution would have required to administer an amount as small as $20 \mu \mathrm{l}$ for an average rat of $250 \mathrm{~g}$. This amount is too small for a proper, definite administration. Therefore the TU oil solution of Andriol Testocaps ${ }^{\circledR}$ was taken out of the capsule and dispersed in Tween 80 solution to obtain a macroemulsion. The main fraction of the oil droplets was in the range 5-10 $\mu \mathrm{m}$. This resembles the dispersion of the oil after release from the capsule which takes place by movement of the gut, the reduced interfacial tension of the gut fluids (approx. $40 \mathrm{mN}$ / $\mathrm{cm}[25])$ and the presence of surfactants such as bile salts. The administration volume of this macroemulsion was $500 \mu$ l. For a rat of $250 \mathrm{~g}$, the emulsion contained $2.5 \mathrm{mg}$ TU and $21 \mathrm{mg}$ oil (lipid). (This lipid was not present when administering the nanosuspensions.)

The maximum serum concentrations $\left(c_{\max }\right)$ obtained with Andriol Testocaps ${ }^{\circledast}$ are half or a little bit more than half of the ones obtained with the nanocrystals. However, Andriol Testocaps ${ }^{\otimes}$ shows a distinctly prolonged serum level. The width of the major serum peak is up to double compared to the nanocrystals. It is assumed that absorption mainly takes place by two mechanisms:

1. The oil droplets are degraded by enzymes, surface-active monoglycerides are formed leading to the formation of TU-loaded micelles. Absorption can take place across the gut wall and via the lymphatic system.

2. The enzymatic degradation is a surface degradation, thus decreasing the size of the TU-loaded oil droplets. When the size is below $100 \mathrm{~nm}$, the droplets are small enough for lymphatic uptake, as described in the literature [26-29]. Potential explanations for the prolonged serum level of Andriol Testocaps ${ }^{\circledR}$ are:

1. For an unknown reason, the oil droplets remain longer in the absorption window.

2. The presence of fat allows absorption at sites where drug from nanocrystals cannot be absorbed anymore. The shape of the observed Andriol Testocaps ${ }^{\circledR}$ serum profile is generally in accordance with the serum profiles published by the group of William Charman. However, it needs to be kept in mind that these studies were done in beagle dogs [2]. Figure 1 (lower) shows the AUC values for the time period of the first 8 hours. Due to the facts explained above, the AUC values for the nanosuspensions are lower than the AUC values of the Andriol formulation. However, taking into account that the nanocrystals were tested without any formulation optimization, the AUC values achieved are very promising. The TU nanosuspension is close to Andriol Testocaps ${ }^{\circledast}$. This holds promise to match the Andriol Testocaps ${ }^{\circledR}$ bioavailability when optimising the TU nanosuspension formulation as outlined above. It seems possible to incorporate a single dose of TU nanocrystals into one tablet or capsule. Instead of optimising the formulation, one could also take the approach to administer a slightly higher dose to compensate for the lower oral bioavailability observed. Such an approach can only be followed in case the "access part of drug" is not absorbed and just passes the gut non-absorbed. In case of TU the problem is that this access drug would be absorbed, immediately be metabolised by the first pass effect of the liver, thus leading to higher TU and T concentrations in the liver which could cause undesired side effects. Therefore it is definitely a smarter approach to optimise the nanocrystals formulation.

Table 2 lists the bioavailability parameters calculated from the serum profiles. The nanocrystals possess higher $c_{\max }$ and shorter $t_{\text {max }^{\prime}}$ which is well described in the literature as a general feature of orally administered nanocrystals [21]. In case such higher $\mathrm{c}_{\max }$ and short $\mathrm{t}_{\max }$ are not required or even should be avoided, the nanocrystals need to be combined with a controlled release technology, e. g. incorporating the nanocrystals into pellets, a hydrocolloid matrix tablet or by coating the single nanocrystals as described by Möschwitzer et al. [30]. There is evidence in literature that the presence of lipid promotes absorption of TU $[\mathbf{1}, \mathbf{2}, \mathbf{4 , 2 3}, \mathbf{2 4}]$, which could be exploited to generally increase the AUC of nanocrystals by co-administration.

\section{BA of Andriol Testocaps ${ }^{\circledast}$ versus Andriol Testocaps ${ }^{\circledR}$ with additional lipid (non-fed vs. fed state)}

Serum concentrations after oral administration of TU are much higher in the fed state compared to the non-fed state. Bagchus et al. found an almost 10 times higher bioavailability in the fed state compared to the fasted state in men [23]. To study the effect of lipid on the absorption, in the second study the bioavailability of Andriol Testocaps ${ }^{\circledR}$ dispersed in Tween 80 solution was compared to Andriol Testocaps ${ }^{\circledast}$ with additionally added lipid, i.e. a mixture of Lauroglycol FCC (Propylene glycol monolaurate ) and castor oil (40\%: $60 \%$ ). 
Muchow et al. Journal of Pharmaceutical Technology \& Drug Research 2013, http://www.hoajonline.com/journals/pdf/2050-120X-2-4.pdf

Table 2. Key pharmacokinetic parameters of the formulations. Explanations are given in the text.

\begin{tabular}{lccr}
\hline Formulation & $\boldsymbol{C}_{\max }[\mathbf{p g} / \mathbf{m l}]$ & $\boldsymbol{T}_{\max }[\mathbf{h o u r s}]$ & AUC after $\mathbf{8} \boldsymbol{h}\left[\mathbf{p} \boldsymbol{g}^{\star} \boldsymbol{h} / \mathbf{m l}\right]$ \\
\hline Andriol in Tween 80 solution ("fasted") & 2,483 & 2 & 8,542 \\
Andriol in castor Oil/Lauroglycol ("fed") & 6,408 & 1 & 13,105 \\
TU Nanosuspension & 3,481 & 2 & 6,667 \\
T Nanosuspension & 3,682 & 1 & 4,312 \\
NLC 30 \% Dynasan (approx. 600 nm) & 5,533 & 1 & 10,208 \\
NLC 30 \% Dynasan (approx. 200 nm) & 6,036 & 1 & 13,950 \\
NLC 15 \% Dynasan (approx. 200 nm) & 8,589 & 1 & 12,933 \\
NLC 15 \% stearic acid (approx. 200 nm) & 6,675 & 2 & 11,976 \\
\hline
\end{tabular}

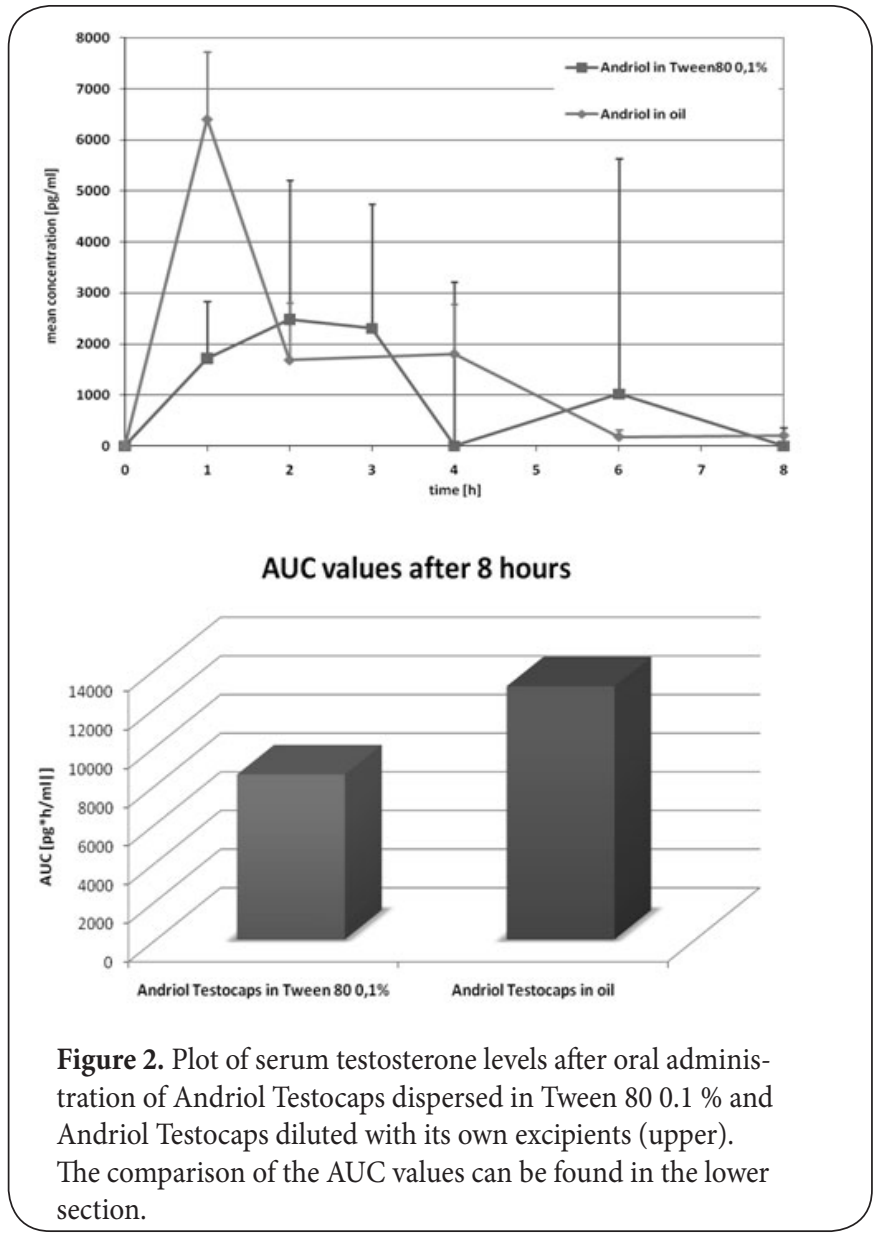

This is exactly the composition of the liquid oil phase in the Andriol Testocaps ${ }^{\circledast}$ capsules. It should be investigated if the presence of more "lipophilic excipient" from the capsule formulation promotes absorption.

Figure 2 (upper) compares the serum profiles of these two formulations. Surprisingly the shape of the serum profiles completely changed when Andriol Testocaps ${ }^{\circledR}$ was administered in an oil phase. The profile resembles very much the profiles obtained with $T$ nanocrystals, a very high $\mathrm{c}_{\max }$ and short $\mathrm{t}_{\max }$. The $\mathrm{c}_{\max }$ is even about $50 \%$ higher compared to the nanocrystals, the $t_{\max }$ is identically at one hour. It cannot be excluded that there would be differences at earlier time points of 15 minutes and 0.5 hours, but this early time points were not covered in these in vivo studies. Clear aim of these studies was to get a first overview, especially to assess if any relevant bioavailability can be obtained at all when administering the developed nanocarriers.

The initial very high serum peak can be explained by better dispersion of the administered oil due to the surfactant present in the oil phase and potential stimulation of bile salt secretion. Both can lead to the formation of finer, smaller oil droplets. Smaller droplets increase the bioavailability as this is well described for the microemulsion formulation of cyclosporine (Sandimmun Neoral, Optoral) [31]. After the initial peak a pronounced tailing of the profile occurs, which does not exist for the investigated nanocrystals. This kind of prolonged serum level is similar to the one of Andriol Testocaps ${ }^{\circledR}$ administered in aqueous Tween solution. It can be summarised that the serum profile obtained from Andriol Testocaps ${ }^{\circledR}$ administered in oil is a superposition of the serum profiles of nanocrystals and Andriol Testocaps ${ }^{\circledast}$ administered in aqueous Tween solution: An initially high serum peak (identical to nanocrystals) followed by a prolonged serum level (identical to Andriol Testocaps ${ }^{\circledR}$ administered dispersed in aqueous surfactant solution).

The serum profiles of the fed state versus the non-fed state in men described in [23] show an even more pronounced change in shape as observed in these studies.

Figure 2 (lower) shows the AUC values of both formulations, the AUC of Andriol Testocaps ${ }^{\circledR}$ in oil is about $50 \%$ higher. It demonstrates the effect of lipid present on the absorption of TU quite well. For a better understanding of the in vivo situation in humans, a model calculation is made. In Andriol Testocaps ${ }^{\circledR}$ capsules, there is $8 \mathrm{mg}$ lipid phase per $1 \mathrm{mg}$ testosterone (total weight of capsule content: $335 \mathrm{mg}$, drug content: $40 \mathrm{mg}$ TU). When dispersing the required dose for the rat of $2.5 \mathrm{mg} / \mathrm{animal} \mathrm{TU}$ in $250 \mathrm{mg}$ oil mixture, this corresponds to $99 \mathrm{mg}$ lipid per $1 \mathrm{mg}$ drug TU. For a dose of $80 \mathrm{mg}$ TU the amount of lipid equals almost $8,000 \mathrm{mg}$. When 


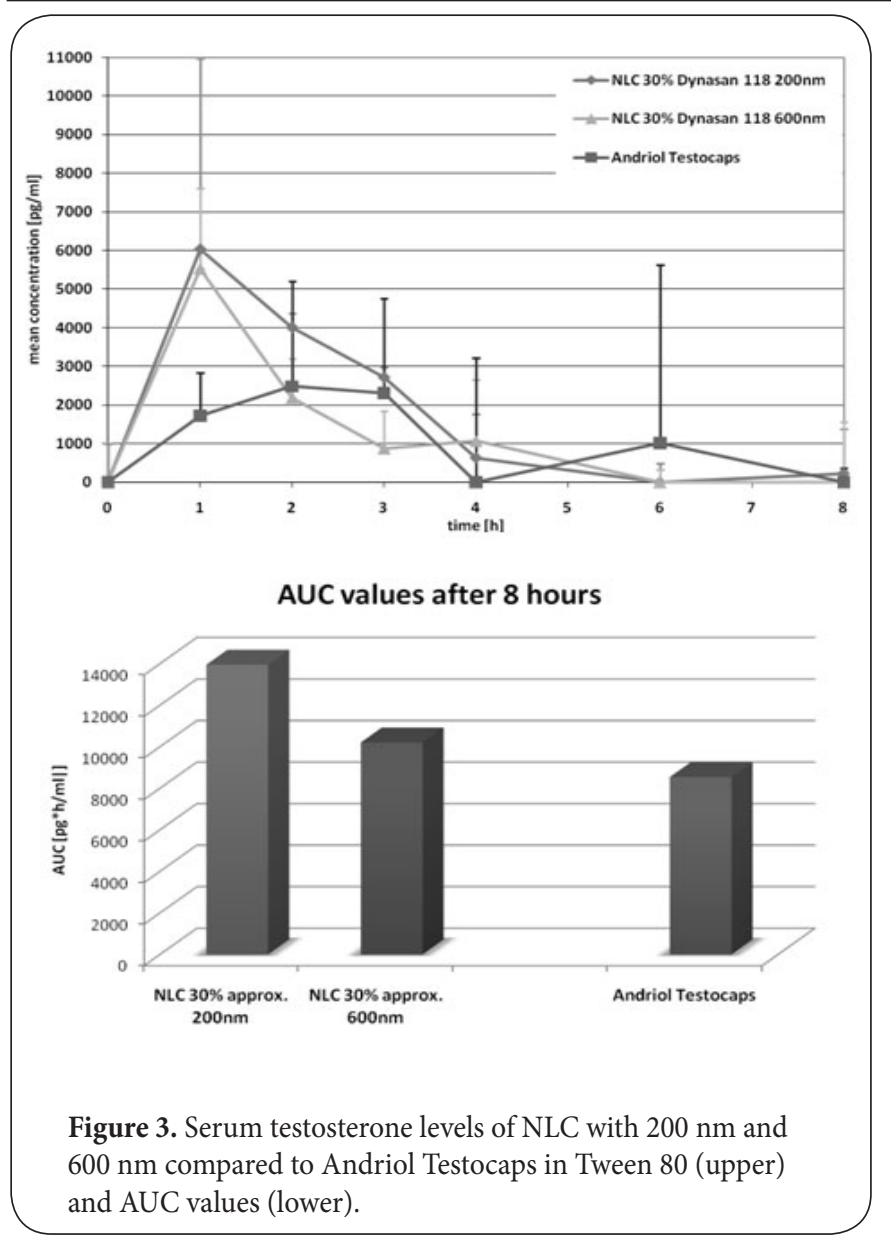

swallowing two capsules of Andriol Testocaps ${ }^{\circledast}, 7.3 \mathrm{~g}$ pure lipid (plus $0.7 \mathrm{~g}$ in the capsules) has to be co-administered to have similar conditions as tested in the animal model. This amount of lipid corresponds to a medium meal of about 100 calories for the lipid alone. Compliance of the patients would probably not be very high if such an amount of additional lipid has to be taken when administering Andriol Testocaps ${ }^{\circledR}$ capsules for $^{\circ}$ improving the oral bioavailability.

Table 2 shows the pharmacokinetic parameters as discussed above, $\mathrm{t}_{\max }$ of Andriol Testocaps ${ }^{\circledR}$ administered in oil are identical to the nanosuspensions of $\mathrm{T}$ but $\mathrm{C}_{\max }$ and $\mathrm{AUC}$ are higher for the Andriol Testocaps ${ }^{\circledR}$ in oil. TU nanosuspension shows a $\mathrm{c}_{\max }$ which is almost equal to the $\mathrm{T}$ nanosuspension but with a later $\mathrm{t}_{\max }$ at $2 \mathrm{~h}$.

\section{Bioavailability of NLC as a function of size $(600 \mathrm{~nm}$ vs. $200 \mathrm{~nm}$ )}

In the next study, two NLC formulations were prepared using the same lipid (Dynasan 118), but with a different size: $600 \mathrm{~nm}$ versus $200 \mathrm{~nm}$. The rationale behind was that the bioavailability of drugs dissolved in oils increases, the better the oil is dispersed in the gut (i. e. the smaller the oil droplet size is). Of course this study could also show whether nanosized lipid particles are superior in bioavailability compared to the oil solution of TU administered in capsules. An improvement was expected based on the data published for cyclosporine [14], when moving to smaller lipid nanoparticles.

Interestingly the two serum profiles of both NLC have an almost identical shape as Andriol Testocaps ${ }^{\circledR}$ administered in oil (= simulation of fed state) (Figure 3, upper). For all the three formulations (NLC $600 \mathrm{~nm}, \mathrm{NLC} 200 \mathrm{~nm}$ and Andriol Testocaps ${ }^{\circledR}$ in oil), $c_{\text {max }}$ and $t_{\text {max }}$ are alike. After the sharp initial peak a prolonged higher serum level occurs, which is most pronounced for the $200 \mathrm{~nm}$ NLC. The serum concentrations of the prolonged level of these $200 \mathrm{~nm}$ particles are up to 1.5-2.0 times higher compared to the $600 \mathrm{~nm} \mathrm{NLC}$. The data prove that NLC administered in a non-fed state exhibit a comparably high bioavailability as TU (Andriol Testocaps ${ }^{\circledR}$ ) administered in oil (resembling the fed state condition). Calculated on a human dose of $80 \mathrm{mg}$ TU, Andriol in fed state corresponded to simultaneous administration of about $8 \mathrm{~g}$ lipid. Administering the $80 \mathrm{mg}$ dose in NLC (30\% drug load) corresponded to co-administration of only about $0.2 \mathrm{mg}$ lipid. Therefore, the administration of an NLC formulation in comparison to the Andriol formulation would be much more patient-friendly and could therefore improve the compliance.

The AUC values depicted in figure $\mathbf{3}$ (lower) show a higher bioavailability of the $600 \mathrm{~nm}$ NLC compared to Andriol Testocaps $^{\circledR}$ (Andriol Testocaps ${ }^{\circledR}$ dispersed in Tween 80 solution). The highest AUC was found for the smaller sized $200 \mathrm{~nm}$ NLC which is in the same range as Andriol Testocaps ${ }^{\circledR}$ in oil (approx. 14,000 units for $200 \mathrm{~nm} \mathrm{NLC,} \mathrm{13,000} \mathrm{units} \mathrm{for}$ Andriol Testocaps ${ }^{\circledast}$ in oil). The pharmacokinetic parameters are given in table 2 .

The conclusions for the study are:

1. The fast onset and initial high serum peak seems to be characteristic for all nanosized formulations (nanocrystals, $\mathrm{NLC}$, very ultra-fine dispersed oil).

2. The total AUC increases with decreasing size of the lipid nanoparticles.

3. Despite that the $200 \mathrm{~nm}$ NLC have a higher total AUC, the initial high serum concentration $\left(c_{\max }\right)$ and $t_{\max }$ of one hour is practically identical. Obviously the reduction in size has little influence on the absolute height of the first initial peak. There seems to be an uptake limitation for NLC or fatty materials.

4. The major difference is that for small sized $200 \mathrm{~nm}$ $\mathrm{NLC}$, there is a higher prolonged serum level. A potential explanation is that the smaller sized NLC are more mucoadhesive. They remain longer in the absorption window in the upper gut, thus yielding higher serum levels. The passage velocity of the $600 \mathrm{~nm}$ NLC seems to be higher, causing less uptake in the absorption window. 5. Another explanation: For lymphatic uptake, the size should be smaller than approx. $100 \mathrm{~nm}$. NLC are surfacedegraded. They reduce in size during passage of the gut. Compared to the $600 \mathrm{~nm} \mathrm{NLC}$, a larger fraction of the 

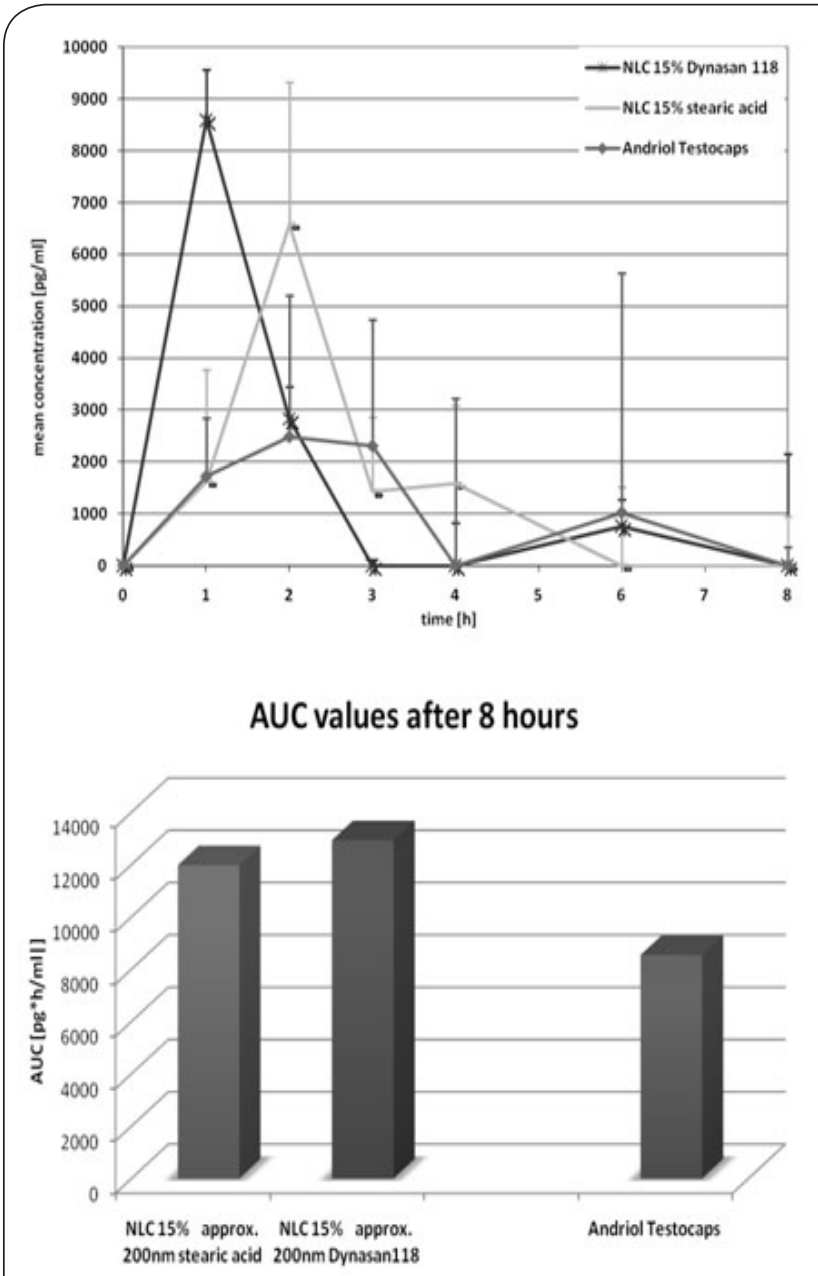

Figure 4. Testosterone serum concentration levels after oral administration of identically sized NLC (200 nm) only differing in the lipid used compared to Andriol Testocaps in Tween 80 (upper) and AUC values (lower).

$200 \mathrm{~nm}$ NLC was sufficiently degraded and reduced in size to be $<100 \mathrm{~nm}$ for lymphatic absorption.

The very high serum concentrations after one hour suggest again that there might be even higher concentrations at 15 minutes and 0.5 hours. This seems possible when the residence time in the stomach is sufficiently short. In contrast to food, stomach emptying happens fast after administration of a liquid (as it was done in this study).

\section{BA of NLC as a function of lipid nature}

In this part of the study, the effect of the chemical nature of the lipid is discussed. The publications by Charman and co-workers report the length of the fatty acids in the oil have a pronounced effect on the extent of absorption of drugs dissolved in such oils. Long-chain fatty acids (C14 to $(18)$ are more effective in promoting absorption than lipids with shorter fatty acid chains [32]. Based on these data two lipids were selected containing $\mathrm{C} 18$ fatty acids. To have additionally a difference in the molecular structure, a triglyceride (Dynasan $118=$ stearic acid triglyceride) was compared with the fatty acid itself (stearic acid).

The serum profiles revealed a very interesting difference (figure 4, upper). The Dynasan NLC exhibit a $c_{\max }$ and $t_{\max }$ identical to all the other nanosized formulations at one hour. However, when using stearic acid as lipid matrix, $\mathrm{t}_{\max }$ moved to two hours. The molecular structure of the lipid has a very distinct effect.

This difference is thought to be associated with the different degradation velocities of the particles and differences in solubilisation of the drug into micelles. In vitro degradation studies with lipase/co-lipase revealed a pronounced dependency of the degradation velocity on the lipid nature (e. g. within various triglycerides/glycerides mixtures, glycerides versus wax). Stearic acid is practically insoluble in water (saturation solubility is $0.3 \mathrm{mg} / 100 \mathrm{~mL}$ ). It is not primarily a substrate for lipases. Triglycerides are degraded by lipases easily. Based on this the size decrease for a Dynasan NLC due to degradation and lipid solubilisation is faster than for the stearic acid NLC. They reach faster a sufficiently small size $(<100 \mathrm{~nm})$ - small enough for lymphatic absorption.

Size decrease by degradation or dissolution of the fatty acid is slower for the stearic acid NLC. However, obviously they stay sufficiently long in the absorption window that a similar bioavailability (AUC) to the Dynasan NLC is reached.

Figure 4 (lower) shows that the AUC for both NLC formulations is practically identical (range of about 12,00013,000 units). To be precise, the AUC is practically identical, but the shape of the serum profiles is different. For the stearic acid $\mathrm{NLC}$, firstly $\mathrm{C}_{\max }$ shifts from one hour to two hours; secondly there is a more pronounced tailing in the decrease of the serum concentration. Serum levels are more prolonged. In contrast to this, a fast decay occurs with Dynasan NLC. After three hours the "absorbable fraction" of the formulation has practically been taken up. The serum concentration was close to 0 . The pharmacokinetic parameters are listed in table $\mathbf{2}$. From the data it can be concluded:

1. It could be confirmed that small NLC $(200 \mathrm{~nm})$ provide highest AUC values.

2. The nature of the lipid matrix seems to have an effect on the absorption kinetics.

3. NLC from lipids which can be fast degraded and/or the lipid with drug which can be solubilised fast, lead to $c_{\max }$ at early times.

4. NLC made from lipids which are slower degraded or solubilised lead to a shift of $c_{\max }$ to a later time point, i.e. increased $t_{\text {max }}$.

5. The similar AUC values obtained suggest that small particles seem to remain sufficiently long in the absorption window (attributed to adhesive properties of the nanoparticles), providing time to be degraded to a adequate small size for lymphatic uptake. 

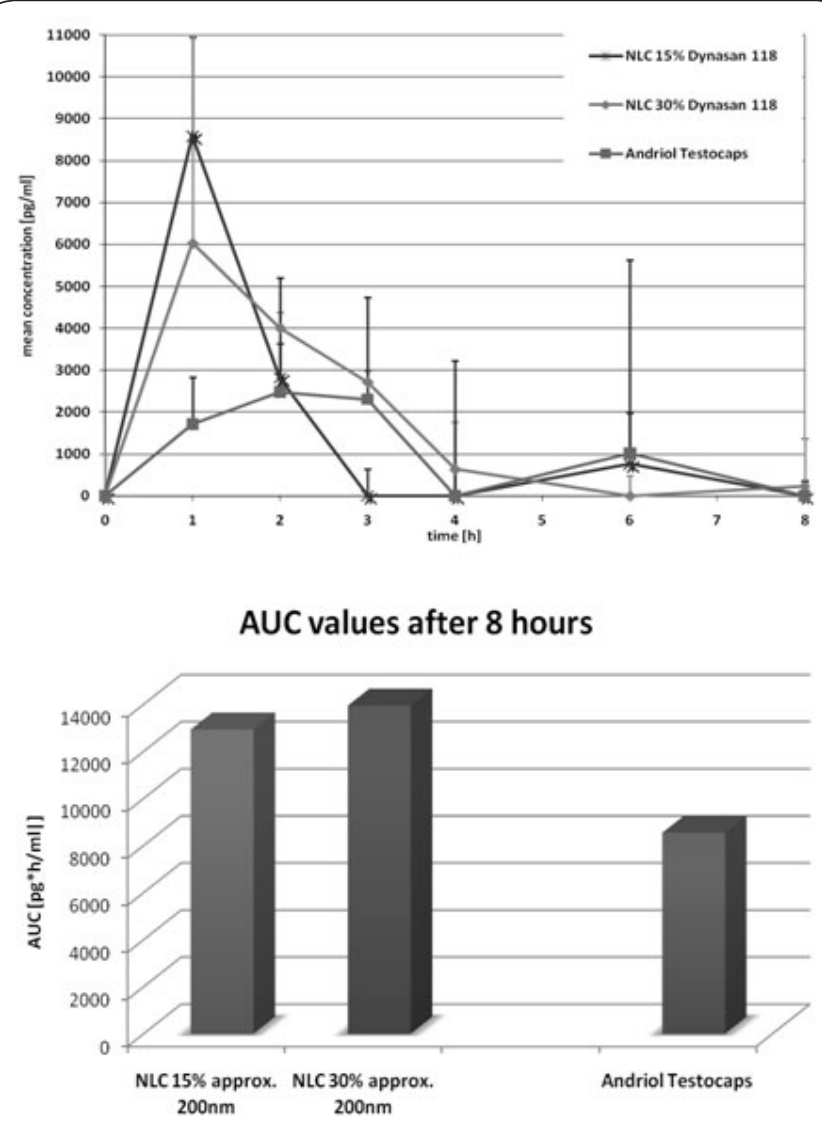

Figure 5. Serum testosterone levels of NLC with $15 \%$ TU and $30 \%$ compared to Andriol Testocaps in Tween 80 (upper) and AUC values (lower).

\section{BA of NLC as a function of lipid: drug ratio}

In a last comparison it was assessed if a larger amount of lipid in the NLC suspensions has an effect on the bioavailability. NLC were prepared with a reduced drug loading (15\% versus $30 \%$ ) that means more NLC needed to be administered for giving the same amount of drug to the animals. The NLC investigated up to this point contained a drug loading of $30 \%$. The particle mass $(=100 \%)$ consisted of $70 \%$ lipid and $30 \%$ drug. The drug loading was reduced to $15 \%$ (NLC particle mass consisted of $85 \%$ lipid and $15 \%$ drug). To administer the same amount of drug, in this case double the number of particles need to be administered. At the same time the amount of lipid per particle was increased (from $70 \%$ to $85 \%)$. When administering the NLC with $15 \%$ TU, an about 2.5 times higher amount of lipid was administered (precisely 2.44 times higher). The total amount of lipid administered to a medium weight rat of $250 \mathrm{~g}$ in a single TU dose of $4 \mathrm{mg}$ was:

$9.3 \mathrm{mg}$ lipid when administering the NLC with $30 \%$ drug loading.

$22.7 \mathrm{mg}$ lipid when administering the NLC with $15 \%$ drug loading.
Looking at the AUC values after eight hours, there is no significant difference between the NLC with $30 \%$ drug load and NLC with $15 \%$ drug load (Figure 5, lower). This appears logic. Even when increasing the amount of lipid by a factor of about 2.5, the total amount of lipid is still very low. Therefore it could not be expected to see such an increase in bioavailability as it was observed when administering Andriol Testocaps ${ }^{\circledast}$ diluted with oil.

However, again very interesting is the difference in the shape of the serum profiles between the two particles (Figure 5, upper). The curves were already plotted in the previous figures, but they are again plotted in one figure together to better illustrate the differences. The NLC with the higher drug load of $30 \%$ show a $c_{\max }$ of about 6,000 at one hour, then a prolonged decay in the serum concentration up to about 4-6 hours. In contrast to this the NLC with $15 \%$ drug load (more lipid per particle) show a $c_{\max }$ of almost 9,000 at one hour, then a sharp decrease to 0 at three hours. From this it can be concluded that the shape of the serum profile can obviously also be modified by changing the drug load of the NLC. It can be imagined that the incorporated drug at a higher concentration slows down the degradation of the particle, thus leading to a slower, prolonged absorption. In case the drug loading is low $(15 \%)$, the degradation velocity is faster, less affected. The particles are fast reduced in size, get below $100 \mathrm{~nm}$ and can be lymphatically taken up. In addition, micelles formed during the particle degradation might contribute to lymphatic uptake.

\section{Conclusions}

The NLC showed a superior ability to increase the bioavailability of TU compared to the commercial product Andriol Testocaps ${ }^{\circledast}$. Reducing the particle size led to higher AUC values, as expected from data, e. g. published with microemulsions. The total AUC with the best formulations was roughly double as high compared to Andriol Testocaps ${ }^{\oplus}$ in non-fed state. Taking into account the reduced volume of the NLC particles compared to Andriol Testocaps ${ }^{\circledR}$ oil solution, administration of a single dose in one oral unit seems to be feasible.

The highest $c_{\max }$ concentrations found with the best formulations were very similar or practically identical. This suggests that there might be a maximum absorption capacity. Absorption might not be further increased, even when further optimising the particles. However, this needs to be verified in additional studies.

Very interesting is that despite the total AUC was similar for the best formulations; the shape of the serum profiles was very different depending on particle characteristics. Main parameters determining the shape of the serum profile are the chemical nature of the lipid matrix (differences in degradation of the particles are assumed) and the drug loading. Lower drug loading and more lipid present (= faster degradation assumed) lead to high $\mathrm{c}_{\max }$ at short $\mathrm{t}_{\max }$ and an 
uptake very fast completed. Higher drug loadings lead also to an early $c_{\max }$ at $t_{\text {max }}$ of one hour, but the $c_{\max }$ is distinctly lower and the decrease in the serum concentration much more prolonged (tailing of profile).

This opens the opportunity of a controlled design of the serum profile by varying the particle characteristics, the nature of the lipid and drug loading (ratio of drug to lipid in the particle). The in vivo data were collected in rats, however the best model (and of course the most expensive) for $\mathrm{T}$ and TU are beagle dogs. Therefore the best formulations screened in this study should be tested in the dog model. To justify such studies, the in vivo animal studies described in this publication provide the first evidence to distinctly increase the bioavailability when using nanocarriers.

\section{Competing interests}

The authors declare that they have no competing interests.

\section{Acknowledgement}

The research work was sponsored by a Galenos Fellowship in the Framework of the EU Project "Towards a European PhD in Advanced Drug Delivery" (Marie Curie Contract MEST CT-2004-404992). In addition, we would like to thank the company PharmaSol GbmH Berlin for scientific support.

\section{Publication history}

Received: 12-Dec-2012 Revised: 24-Dec-2012

Accepted: 07-Jan-2013 Published: 12-Jan-2013

\section{References}

1. Kohn FM, Schill WB: A new oral testosterone undecanoate formulation. World J Urol 2003, 21:311-315. | Article | PubMed

2. Shackleford DM, Faassen WA, Houwing N, Lass H, Edwards GA, Porter CJ, Charman WN: Contribution of lymphatically transported testosterone undecanoate to the systemic exposure of testosterone after oral administration of two andriol formulations in conscious lymph ductcannulated dogs. J Pharmacol Exp Ther 2003, 306:925-933. | Article | PubMed

3. Horst HJ, Erdmann T: Recovery of free androgens in the rat prostate in vivo and in vitro after treatment with orally active testosterone undecanoate (TU). Horm Metab Res 1980, 12:541-545. | Article | PubMed

4. Horst HJ, Holtje WJ, Dennis M, Coert A, Geelen J, Voigt KD: Lymphatic absorption and metabolism of orally administered testosterone undecanoate in man. Klin Wochenschr 1976, 54:875-879. | Article | PubMed

5. Houwing NS, Maris F, Schnabel PG, Bagchus WM: Pharmacokinetic study in women of three different doses of a new formulation of oral testosterone undecanoate, Andriol Testocaps. Pharmacotherapy 2003, 23:1257-1265. | Article | PubMed

6. W.O. Foye, T.L. Lemke, D.A. Williams: Foye's principles of medicinal chemistry, Lippincott Williams \& Wilkins, 2007.

7. Frey $H$, Aakvaag A, Saanum D, Falch J: Bioavailability of oral testosterone in males. Eur J Clin Pharmacol 1979, 16:345-349. | Article I PubMed

8. R.H. Müller, K. Mäder, A. Lippacher, V. Jenning, Fest-flüssige (halbfeste) Lipidpartikel und Verfahren zur Herstellung hochkonzentrierter LipidpartikeldispersionenPCT/EP00/045652000.

9. Muchow $M$, Maincent $P$, Müller RH: Lipid nanoparticles with a solid matrix (SLN, NLC, LDC) for oral drug delivery. Drug Dev Ind Pharm 2008, 34:1394-1405. | Article | PubMed
10. R.H. Müller, Zubereitung in Form eines wahlweise wirkstoffhaltigen Matrix-Hilfsstoff Compounds (Preparation in form of a matrix materialauxiliary agent compound containing optionally an active substance), European Union Patent PCT/EP97/06893 (P 47025)1997.

11. Mehnert W, Mäder K: Solid lipid nanoparticles: production, characterization and applications. Adv Drug Deliv Rev 2001, 47:165-196. | Article | PubMed

12. R.H. Müller, C. Schwarz, A. zur Mühlen, W. Mehnert, Incorporation of lipophilic drugs and drug release profiles of solid lipid nanoparticles (SLN). Int. Symp. Control. Release Bioact. Mater. 21, 1994, pp. 146-147.

13. M.R. Gasco, Method for producing solid lipid microspheres having a narrow size distribution, USA Patent 5, 250, 2361993.

14. Müller RH, Runge S, Ravelli V, Mehnert W, Thunemann AF, Souto EB: Oral bioavailability of cyclosporine: solid lipid nanoparticles (SLN) versus drug nanocrystals. Int J Pharm 2006, 317:82-89. | Article | PubMed

15. R.H. Müller, S.A. Runge, V. Ravelli, Pharmaceutical Cyclosporin Formulation of improved Biopharmaceutical Performance and Improved Physical Quality and Stability and Process for Producing Same. Germany Patent DE 19819273 A11998.

16. Müller RH, Runge SA, Ravelli V, Thünemann AF, Mehnert W, Souto EB: Cyclosporine-loaded solid lipid nanoparticles (SLN $\left.{ }^{\circledR}\right)$ : Druglipid physicochemical interactions and characterization of drug incorporation. European Journal of Pharmaceutics and Biopharmaceutics 2008, 68:535-544. | Article

17. L. Penkler, R.H. Müller, S. Runge, V. Ravelli, Pharmaceutical cyclosporin formulation with improved biopharmaceutical properties, improved physical quality and greater stability, and method for producing said formulation, European Union Patent PCT application PCT/ EP99/028921999.

18. L. Penkler, R.H. Müller, S. Runge, V. Ravelli, Pharmaceutical cyclosporin formulation with improved biopharmaceutical properties, improved physical quality and greater stability, and method for producing said formulation, USA Patent 6,551,6192003.

19. Keck CM, Müller RH: Drug nanocrystals of poorly soluble drugs produced by high pressure homogenisation. Eur J Pharm Biopharm 2006, 62:3-16. | Article | PubMed

20. Moschwitzer J, Müller RH: New method for the effective production of ultrafine drug nanocrystals. J Nanosci Nanotechnol 2006, 6:3145-3153. | Article | PubMed

21. Liversidge GG, Cundy KC: Particle size reduction for improvement of oral bioavailability of hydrophobic drugs: I. Absolute oral bioavailability of nanocrystalline danazol in beagle dogs. International Journal of Pharmaceutics 1995, 125:91-97. | Article

22. J. Möschwitzer, R.H. Müller, in: D. Thassu, M. Deleers and Y. Pathak (Eds.), Nanoparticulate Drug Delivery Systems: Recent Trends and Emerging Technologies, Informa Healthcare, 2007.

23. Bagchus WM, Hust R, Maris F, Schnabel PG, Houwing NS: Important effect of food on the bioavailability of oral testosterone undecanoate. Pharmacotherapy 2003, 23:319-325. | Article | PubMed

24. Schnabel PG, Bagchus W, Lass H, Thomsen T, Geurts TB: The effect of food composition on serum testosterone levels after oral administration of Andriol Testocaps. Clin Endocrinol (Oxf) 2007, 66:579585. | Article | PubMed Abstrct | PubMed Full Text

25. Kalantzi L, Goumas K, Kalioras V, Abrahamsson B, Dressman JB, Reppas C: Characterization of the human upper gastrointestinal contents under conditions simulating bioavailability/bioequivalence studies. Pharm Res 2006, 23:165-176. | Article | PubMed

26. R.H. Müller, C. Olbrich: Solid Lipid Nanoparticles (SLN): Phagocytic uptake, in vitro cytotoxity and in vivo biodegradation. Drugs 1999 42:65-88.

27. C. Olbrich, Mehnert, W., Müller, R.H., In vitro Degradation Properties of Solid Lipid Nanoparticles SLN ${ }^{\mathrm{TM}}$. 2nd World Meeting on Pharmaceutics, Biopharmaceutics, Pharmaceutical Technology, Paris, 1998, pp. 577-578.

28. Olbrich C, Kayser O, Müller RH: Lipase degradation of Dynasan 114 and 116 solid lipid nanoparticles (SLN)--effect of surfactants, storage time 
Muchow et al. Journal of Pharmaceutical Technology \& Drug Research 2013,

http://www.hoajonline.com/journals/pdf/2050-120X-2-4.pdf

and crystallinity. Int J Pharm 2002, 237:119-128. | Article | PubMed

29. Olbrich C, Müller RH: Enzymatic degradation of SLN-effect of surfactant and surfactant mixtures. Int J Pharm 1999, 180:31-39. | Article | PubMed

30. Moschwitzer J, Müller RH: Spray coated pellets as carrier system for mucoadhesive drug nanocrystals. Eur J Pharm Biopharm 2006, 62:282287. I Article | PubMed

31. A. Meinzer, E. Müller, J. Vonderscher, in: R. H. Müller and G. E. Hildebrand (Eds.), Pharmazeutische Technologie: Moderne Arzneiformen, Wissenschaftliche Verlagsgesellschaft, Stuttgart, 1998, Vol. 2:169-177.

32. Porter CJ, Charman WN: Lipid-based formulations for oral administration: opportunities for bioavailability enhancement and lipoprotein targeting of lipophilic drugs. J Recept Signal Transduct Res 2001, 21:215-257. | Article | PubMed

\section{Citation:}

Muchow M, Maincent P, Müller R H and Keck C M: Testosterone undecanoate - increase of oral bioavailability by nanostructured lipid carriers (NLC). journal of Pharmaceutical Technology and Drug Research 2013, 2:4.

http://dx.doi.org/10.7243/2050-120X-2-4 